\title{
Absorption Time of the Moran Process*
}

\author{
Josep Díaz ${ }^{1}$, Leslie Ann Goldberg ${ }^{2}$, David Richerby ${ }^{2}$, and \\ Maria Serna ${ }^{1}$
}

1 Departament de Llenguatges i Sistemes Informàtics, Universitat Politècnica de Catalunya, Spain

2 Department of Computer Science, University of Oxford, Wolfson Building, Parks Road, Oxford, OX1 3QD, UK

\begin{abstract}
The Moran process models the spread of mutations in populations on graphs. We investigate the absorption time of the process, which is the time taken for a mutation introduced at a randomly chosen vertex to either spread to the whole population, or to become extinct. It is known that the expected absorption time for an advantageous mutation is $O\left(n^{4}\right)$ on an $n$-vertex undirected graph, which allows the behaviour of the process on undirected graphs to be analysed using the Markov chain Monte Carlo method. We show that this does not extend to directed graphs by exhibiting an infinite family of directed graphs for which the expected absorption time is exponential in the number of vertices. However, for regular directed graphs, we show that the expected absorption time is $\Omega(n \log n)$ and $O\left(n^{2}\right)$. We exhibit families of graphs matching these bounds and give improved bounds for other families of graphs, based on isoperimetric number. Our results are obtained via stochastic dominations which we demonstrate by establishing a coupling in a related continuous-time model. The coupling also implies several natural domination results regarding the fixation probability of the original (discrete-time) process, resolving a conjecture of Shakarian, Roos and Johnson.
\end{abstract}

1998 ACM Subject Classification F.2.2 Nonnumerical Algorithms and Problems

Keywords and phrases Moran Process

Digital Object Identifier 10.4230/LIPIcs.APPROX-RANDOM.2014.630

\section{Introduction}

The Moran process [22], as adapted by Lieberman, Hauert and Nowak [17], is a stochastic model for the spread of genetic mutations through populations of organisms. Similar processes have been used to model the spread of epidemic diseases, the behaviour of voters, the spread of ideas in social networks, strategic interaction in evolutionary game theory, the emergence of monopolies, and cascading failures in power grids and transport networks $[2,3,12,16,18]$.

In the Moran process, individuals are modelled as the vertices of a graph and, at each step of the discrete-time process, an individual is selected at random to reproduce. This vertex chooses one of its neighbours uniformly at random and replaces that neighbour with its offspring, a copy of itself. The probability that any given individual is chosen to reproduce is proportional to its fitness: individuals with the mutation have fitness $r>0$ and non-mutants have fitness 1 . The initial state has a single mutant placed uniformly at random in the graph,

* The research leading to these results has received funding from the European Research Council under the European Union's Seventh Framework Programme (FP7/2007-2013) ERC grant agreement no. 334828.

The paper reflects only the authors' views and not the views of the ERC or the European Commission.

The European Union is not liable for any use that may be made of the information contained therein.

cc) (i) Josep Díaz, Leslie Ann Goldberg, David Richerby, and Maria Serna

cc. licensed under Creative Commons License CC-BY

17th Int'l Workshop on Approximation Algorithms for Combinatorial Optimization Problems (APPROX'14) /

18th Int'l Workshop on Randomization and Computation (RANDOM'14).

Editors: Klaus Jansen, José Rolim, Nikhil Devanur, and Cristopher Moore; pp. 630-642

Leibniz International Proceedings in Informatics
LIPICS Schloss Dagstuhl - Leibniz-Zentrum für Informatik, Dagstuhl Publishing, Germany 
with every other vertex a non-mutant. On any finite, strongly connected graph, the process will terminate with probability 1 , either in the state where every vertex is a mutant (known as fixation) or in the state where no vertex is a mutant (known as extinction).

The principal quantities of interest are the fixation probability (the probability of reaching fixation) and the expected absorption time (the expected number of steps before fixation or extinction is reached). In general, these depend on both the graph topology and the mutant fitness. In principle, they can be computed by standard Markov chain techniques but doing so for an $n$-vertex graph involves solving a set of $2^{n}$ linear equations, which is computationally infeasible. Fixation probabilities have also been calculated by producing and approximately solving a set of differential equations that model the process [14]. These methods seem to work well in practice but there is no known bound on the error introduced by converting to differential equations and approximating their solution.

When the underlying graph is undirected and the mutant fitness is $r>1$, there is a fully polynomial randomised approximation scheme (FPRAS) for computing the fixation probability [10]. The FPRAS uses the Markov chain Monte Carlo method and provides a suitable approximation in polynomial time because the expected absorption time on an $n$-vertex graph is at most $\frac{r}{r-1} n^{4}$ for $r>1$.

\subsection{Our Contributions}

The main contribution of this paper is to determine the extent to which the polynomial bound on expected absorption time carries through to directed graphs. Throughout the paper, we assume that the mutant fitness $r$ exceeds 1.

\subsubsection{Regular Digraphs}

We start by considering the absorption time on a strongly connected $\Delta$-regular digraph (where every vertex has in-degree $\Delta$ and out-degree $\Delta$ ). Regularity makes some calculations straightforward because the detailed topology of the graph is not relevant. We describe these first, and then discuss the more difficult questions (where topology does play a role) and state our results.

The following facts hold for $\Delta$-regular graphs, independent of the topology.

- It is well known [17] that the fixation probability of any regular $n$-vertex graph is

$$
\frac{1-r^{-1}}{1-r^{-n}}
$$

To see this, note that if there are $k$ mutants, the total fitness of the population is $W_{k}=n+k(r-1)$. The probability that the next reproduction happens along the directed edge $(u, v)$ is $\frac{r}{W_{k}} \frac{1}{\Delta}$ if $u$ is a mutant and $\frac{1}{W_{k}} \frac{1}{\Delta}$, if it is not. Since the graph is $\Delta$-regular, there are exactly as many directed edges from mutants to non-mutants as there are from non-mutants to mutants. Thus, the probability that the number of mutants increases at the next step is exactly $r$ times the probability that it decreases, regardless of which vertices are currently mutants. Thus, the number of mutants in the population, observed every time it changes, forms a random walk on $\{0, \ldots, n\}$ with initial state 1 , upward drift $r$ and absorbing barriers at 0 and $n$. It is standard (e.g., [13, Example 3.9.6]) that such a random walk reaches $n$ with the probability given by (1).

- It is also well known (e.g., [13, Example 3.9.6] or, for an approximation, [23, eq. (13)]) that the expected number of steps of this walk before absorption (which may be at either 0 or $n$ ) is a function of $r$ and $n$ that tends to $n\left(1+\frac{1}{r}\right)$ in the limit as $n \rightarrow \infty$, independent 
of the graph structure beyond regularity. However, the number of steps taken by the random walk (often referred to as the "active steps" of the Moran process) is not the absorption time of the original process, which includes many steps at which the number of mutants does not change, either because a mutant reproduces to a mutant or because a non-mutant reproduces to a non-mutant.

In Section 4, we show that the expected absorption time of the Moran process is polynomial for regular digraphs. In contrast to the number of active steps, the absorption time does depend on the detailed structure of the graph. We prove the following upper and lower bounds, where $H_{n}$ denotes the $n^{\prime}$ th harmonic number, which is $\Theta(\log n)$.

- Theorem 1. The expected absorption time of the Moran process on a strongly connected $\Delta$-regular $n$-vertex digraph $G$ is at least $\left(\frac{r-1}{r^{2}}\right) n H_{n-1}$ and at most $n^{2} \Delta$.

In Section 4.5, we prove the following theorem, which shows that the upper bound in Theorem 1 is tight up to a constant factor (which depends on $\Delta$ and $r$ but not on $n$ ).

- Theorem 2. Suppose that $r>1$ and $\Delta>2$. There is an infinite family $\mathcal{G}$ of $\Delta$-regular graphs such that, when the Moran process is run on an n-vertex graph $G \in \mathcal{G}$, the expected absorption time exceeds $\frac{1}{8 r}\left(1-\frac{1}{r}\right) \frac{n^{2}}{(\Delta-1)^{2}}$.

The digraphs in the family $\mathcal{G}$ are symmetric, so can be viewed as undirected graphs. The upper bound on the expected absorption time in Theorem 1 can be improved for certain classes of regular undirected graphs using the notion of the isoperimetric number $i(G)$ of a graph $G$, which is defined in Section 4.4. In the full version, we prove the following theorem.

- Theorem 3. The expected absorption time of the Moran process on a connected $\Delta$-regular $n$-vertex undirected graph $G$ is at most $2 \Delta n H_{n} / i(G)$.

Theorem 3 pinpoints the expected absorption time for $G=K_{n}$, up to a constant factor, since $i\left(K_{n}\right)=\lceil n / 2\rceil[21]$ and Theorem 1 gives an $\Omega(n \log n)$ lower bound. Theorem 3 is worse than the upper bound of Theorem 1 by a factor of $O(\log n)$ for the cycle $C_{n}$ since $i\left(C_{n}\right)=$ $2 /\lfloor n / 2\rfloor[21]$. However, we often get an improvement by using the isoperimetric number. For example, the $\sqrt{n}$-by- $\sqrt{n}$ grid has $i(G)=\Theta(1 / \sqrt{n})$ (see [8]), giving an $O\left(n^{3 / 2} \log n\right)$ absorption time; the hypercube has $i(G)=1$ (see, e.g., [21]), giving an $O\left(n \log ^{2} n\right)$ absorption time. Bollobás [4] showed that, for every $\Delta \geq 3$ there is a positive number $\eta<1$ such that, for almost all $\Delta$-regular $n$-vertex undirected graphs $G$ (as $n$ tends to infinity), $i(G) \geq(1-\eta) \Delta / 2$, which gives an $O(n \log n)$ absorption time since these graphs are connected.

\subsubsection{Slow Absorption}

Theorem 1 shows that regular digraphs, like undirected graphs, reach absorption in expected polynomial time. In Section 5 we show that the same does not hold for general digraphs. In particular, we construct an infinite family $\left\{G_{r, N}\right\}$ of strongly connected digraphs indexed by a positive integer $N$. We then prove the following theorem.

- Theorem 4. Fix $r>1$ and let $\varepsilon_{r}=\min (r-1,1)$. For any positive integer $N$, large enough with respect to $r$, the expected absorption time of the Moran process on $G_{r, N}$ is at least

$$
\frac{1}{16}\left\lfloor\left(\frac{\varepsilon_{r}}{32}\right)\left(2^{N}-1\right)\right\rfloor \frac{\varepsilon_{r}}{4\lceil r\rceil+3} .
$$


Theorem 4 shows that there is an infinite family of strongly connected digraphs in which absorption time of an $n$-vertex graph is exponentially large, as a function of $n$. Thus, the techniques from [10] do not give a polynomial-time algorithm for approximating the fixation probability on digraphs.

The underlying structure of the graph $G_{r, N}$ is a large clique on $N$ vertices and a long directed path. Each vertex of the clique sends an edge to the first vertex of the path, and each vertex of the clique receives an edge from the path's last vertex. We refer to the first $N$ vertices of path as $P$ and the remainder as $Q$. Each vertex of $P$ has out-degree 1 but receives $4\lceil r\rceil$ edges from $Q$. (See Figure 1.)

Suppose that $N$ is sufficiently large with respect to $r$ and consider the Moran process on $G_{r, N}$. There is a reasonable probability (about $\frac{1}{4 r+2}$ ) that the initial mutant is in the clique. The edges to and from the path have a negligible effect so it is reasonably likely (probability at least $1-\frac{1}{r}$ ) that we will then reach the state where half the clique vertices are mutants. To reach absorption from this state, one of two things must happen.

For the process to reach extinction, the mutants already in the clique must die out. Because the interaction between the clique and path is small, the number of mutants in the clique is very close to a random walk on $\{0, \ldots, N\}$ with upward drift $r$, and the expected time before such a walk reaches zero from $N / 2$ is exponential in $N$.

On the other hand, suppose the process reaches fixation. In particular, the vertices of $P$, the first part of the path, must become mutants. Note that no vertex of $Q$ can become a mutant before the last vertex of $P$ has done so. While all the vertices in $Q$ are non-mutants, the edges from that part of the path to $P$ ensure that each mutant in $P$ is more likely to be replaced by a non-mutant from $Q$ than it is to create a new mutant in $P$. As a result, the number of mutants in $P$ is bounded above by a random walk on $\{0, \ldots, N\}$ with a strictly greater probability of decreasing than increasing. Again, this walk is expected to take exponentially many steps before reaching $N$.

\subsubsection{Stochastic Domination}

Our main technical tool is stochastic domination. Intuitively, one expects that the Moran process has a higher probability of reaching fixation when the set of mutants is $S$ than when it is some subset of $S$, and that it is likely to do so in fewer steps. It also seems obvious that modifying the process by continuing to allow all transitions that create new mutants but forbidding some transitions that remove mutants should make fixation faster and more probable. Such intuitions have been used in proofs in the literature; it turns out that they are essentially correct, but for rather subtle reasons.

The Moran process can be described as a Markov chain $\left(Y_{t}\right)_{t \geq 1}$ where $Y_{t}$ is the set $S \subseteq V(G)$ of mutants at the $t^{\prime}$ th step. The normal method to make the above intuitions formal would be to demonstrate a stochastic domination by coupling the Moran process $\left(Y_{t}\right)_{t \geq 1}$ with another copy $\left(Y_{t}^{\prime}\right)_{t \geq 1}$ of the process where $Y_{1} \subseteq Y_{1}^{\prime}$. The coupling would be designed so that $Y_{1} \subseteq Y_{1}^{\prime}$ would ensure that $Y_{t} \subseteq Y_{t}^{\prime}$ for all $t>1$. However, a simple example shows that such a coupling does not always exist for the Moran process. Let $G$ be the undirected path with two edges: $V(G)=\{1,2,3\}$ and $E(G)=\{(1,2),(2,1),(2,3),(3,2)\}$. Let $\left(Y_{t}\right)_{t \geq 1}$ and $\left(Y_{t}^{\prime}\right)_{t \geq 1}$ be Moran processes on $G$ with $Y_{1}=\{2\}$ and $Y_{1}^{\prime}=\{2,3\}$. With probability $\frac{r}{2(r+2)}$, we have $Y_{2}=\{1,2\}$. The only possible value for $Y_{2}^{\prime}$ that contains $Y_{2}$ is $\{1,2,3\}$ but this occurs with probability only $\frac{r}{2(2 r+1)}$. Therefore, any coupling between the two processes fails because

$$
\operatorname{Pr}\left(Y_{2} \not \subset Y_{2}^{\prime}\right) \geq \frac{r(r-1)}{2(r+2)(2 r+1)},
$$


which is strictly positive for any $r>1$. The problem is that, when vertex 3 becomes a mutant, it becomes more likely to be the next vertex to reproduce and, correspondingly, every other vertex becomes less likely. This can be seen as the new mutant "slowing down" all the other vertices.

To get around this problem, we consider a continuous-time version of the process, $\widetilde{Y}[t]$ $(t \geq 0)$. Given the set of mutants $\widetilde{Y}[t]$ at time $t$, each vertex waits an amount of time before reproducing. For each vertex, this period of time is chosen according to the exponential distribution with parameter equal to the vertex's fitness, independently of the other vertices. (Thus, the parameter is $r$ if the vertex is a mutant and 1, otherwise.) If the first vertex to reproduce is $v$ at time $t+\tau$ then, as in the standard, discrete-time version of the process, one of its out-neighbours $w$ is chosen uniformly at random, the individual at $w$ is replaced by a copy of the one at $v$ and the time at which $w$ will next reproduce is exponentially distributed with parameter given by its new fitness. The discrete-time process is recovered by taking the sequence of configurations each time a vertex reproduces.

In continuous time, each member of the population reproduces at a rate given by its fitness, independently of the rest of the population whereas, in discrete time, the population has to co-ordinate to decide who will reproduce next. It is still true in continuous time that vertex $w$ becoming a mutant makes it less likely that each vertex $v \neq w$ will be the next to reproduce. However, the vertices are not slowed down as they are in discrete time: they continue to reproduce at rates determined by their fitnesses. This distinction allows us to establish the following coupling lemma, which formalises the intuitions discussed above.

- Lemma 5 (Coupling lemma). Let $G=(V, E)$ be any digraph, let $Y \subseteq Y^{\prime} \subseteq V(G)$ and $1 \leq r \leq r^{\prime}$. Let $\tilde{Y}[t]$ and $\widetilde{Y}^{\prime}[t](t \geq 0)$ be continuous-time Moran processes on $G$ with mutant fitness $r$ and $r^{\prime}$, respectively, and with $\widetilde{Y}[0]=Y$ and $\widetilde{Y}^{\prime}[0]=Y^{\prime}$. There is a coupling between the two processes such that $\widetilde{Y}[t] \subseteq \widetilde{Y}^{\prime}[t]$ for all $t \geq 0$.

In the paper, we use the coupling lemma to establish stochastic dominations between discrete Moran processes. It also has consequences concerning fixation probabilities. The fixation probability $f_{G, r}$ is the probability that the all-mutant state is reached when the Moran process is run on a digraph $G=(V, E)$, starting from a state in which a single initial mutant is placed uniformly at random. For any set $S \subseteq V$, let $f_{G, r}(S)$ be the probability of reaching fixation when the set of vertices initially occupied by mutants is $S$. Thus, $f_{G, r}=\frac{1}{|V|} \sum_{v \in V} f_{G, r}(\{v\})$. Using the coupling lemma, we can prove the following theorem.

- Theorem 6. For any digraph $G$, if $0<r \leq r^{\prime}$ and $S \subseteq S^{\prime} \subseteq V(G)$, then $f_{G, r}(S) \leq$ $f_{G, r^{\prime}}\left(S^{\prime}\right)$.

This theorem has two immediate corollaries. The first was conjectured by Shakarian, Roos and Johnson [26, Conjecture 2.1]. It was known from [25] that $f_{G, r} \geq f_{G, 1}$ for any $r>1$.

- Corollary 7 (Monotonicity). If $0<r \leq r^{\prime}$ then, for any digraph $G, f_{G, r} \leq f_{G, r^{\prime}}$.

Corollary 7 follows immediately from Theorem 6 since $f_{G, r}(\{v\}) \leq f_{G, r^{\prime}}(\{v\})$ for all $v \in V(G)$.

The second corollary says that adding more mutants can't decrease the fixation probability and has been assumed in the literature, without proof. However, although it appears obvious at first, it is somewhat subtle: we have seen that adding more mutants can actually decrease the probability of a particular vertex becoming a mutant at the next step of the process.

- Corollary 8 (Subset domination). For any digraph $G$ and any $r>0$, if $S \subseteq S^{\prime} \subseteq V(G)$, then $f_{G, r}(S) \leq f_{G, r}\left(S^{\prime}\right)$. 
Note that, although we have introduced the continuous-time version of the process for technical reasons, to draw conclusions about the original, discrete-time Moran process, the continuous-time version may actually be a more realistic model than the discrete-time version.

\subsection{Previous Work}

Fixation probabilities in the Moran process are known for regular graphs [17] and stars (complete bipartite graphs $K_{1, k}$ ) [6]. Lieberman et al. [17] give classes of directed graphs with a parameter $k$, and claim that the fixation probability on these graphs tends to $1-r^{-k}$ for large graphs. While these graphs do seem to have very large fixation probability, we have shown this specific claim to be incorrect for $k=5$ [9]. Very recently, it has been claimed [15] that taking $k=\Theta\left(n^{1 / 6}\right)$ does yield fixation probability tending to 1 (as a function of $n$, independently of $r$ ) but no rigorous proof is given. Other work has investigated the possibility of so-called "suppressors", graphs having fixation probability less than that given by (1) for at least some range of values for $r[19,20]$.

There is a more complicated version of the Moran process in which the fitness of a vertex is determined by its expected payoff when playing some two-player game against a randomly chosen neighbour $[27,26]$. In this version of the process, mutants play the game with one strategy and non-mutants play the game with another. The ordinary Moran process corresponds to the special case of this game in which the mutant and non-mutant strategies give payoffs $r$ and 1, respectively, regardless of the strategy used by the opponent.

Most previous work on absorption times has been in the game-based version of the process, where the added complexity of the model limits analysis to very simple graphs, such as complete graphs, stars and cycles $[1,5]$.

\section{Preliminaries}

When $k$ is a positive integer, $[k]$ denotes $\{1, \ldots, k\}$. We consider the evolution of the Moran process [17] on a strongly connected directed graph (digraph). Consider such a digraph $G=(V, E)$ with $n=|V|$. When the process is run on $G$, each state is a set of vertices $S \subseteq V$. The vertices in $S$ are said to be "mutants". If $|S|=k$ then the total fitness of the state is given by $W_{k}=n+(r-1) k$ - each of the $k$ mutants contributes fitness $r$ to the total fitness and each other vertex contributes fitness 1. Except where stated otherwise, we assume that $r>1$. The starting state is chosen uniformly at random from the one-element subsets of $V(G)$. From a state $S$ with $|S|=k$, the process evolves as follows. First, a vertex $u$ is chosen to reproduce. The probability that vertex $u$ is chosen is $r / W_{k}$ if $u$ is a mutant and $1 / W_{k}$ otherwise. Given that $u$ will reproduce, a directed edge $(u, v)$ is chosen uniformly at random from $\left\{\left(u, v^{\prime}\right) \mid\left(u, v^{\prime}\right) \in E\right\}$. The state of vertex $u$ in $S$ is copied to $v$ to give the new state, which is $S \cup\{v\}$ if $u$ is a mutant and $S \backslash\{v\}$, otherwise.

A digraph $G=(V, E)$ is $\Delta$-regular if every vertex has in- and out-degree $\Delta$. G is regular if it is $\Delta$-regular for some natural number $\Delta$. If the Moran process is run on a strongly connected digraph $G$, there are exactly two absorbing states - $\emptyset$ and $V(G)$. Once one of these states is reached, the process will stay in it forever. The absorption time is the number of steps until such a state is reached.

A digraph $G=(V, E)$ is weakly connected if the underlying undirected graph is connected. Given a subset $S \subseteq V$, let $m_{S}^{+}$be the number of edges from vertices in $S$ to vertices in $V \backslash S$. Let $m_{S}^{-}$be the number of edges from vertices in $V \backslash S$ to $S$. Note that every regular digraph that is weakly connected is strongly connected. 
We sometimes consider the Moran process on an undirected graph $G=(V, E)$. We view the undirected graph as a digraph in which the set $E$ of edges is symmetric (so $(u, v) \in E$ if and only if $(v, u) \in E)$.

All proofs are included in the full version, which is available at [11]. To assist the reader, we have used the numbering from the full version in the extended abstract (so there are gaps in the numbering sequence here).

\section{Domination}

A useful proof technique is to show that the behaviour of the Moran process is stochastically dominated by that of a related process that is easier to analyse. Similarly, it is useful to compare the behaviour of the Moran process, evolving on a digraph $G$, with that of another Moran process on the same digraph, where the second process starts with more mutants. Recall that the Moran process can be described as a Markov chain $\left(Y_{t}\right)_{t \geq 1}$ where $Y_{t}$ is the set $S \subseteq V(G)$ of mutants at the $t^{\prime}$ th step. It would be natural to attempt to establish a coupling between Moran processes $\left(Y_{t}\right)_{t \geq 1}$ and $\left(Y_{t}^{\prime}\right)_{t \geq 1}$ such that, if $Y_{1} \subseteq Y_{1}^{\prime}$, then $Y_{t} \subseteq Y_{t}^{\prime}$ for all $t \geq 1$, but this cannot be done, as shown in Section 1.1.3.

To obtain useful dominations, we will consider a continuous-time version of the Moran process. The domination that we construct for the continuous-time process will allow us to draw conclusions about the original (discrete-time) Moran process. In a digraph $G=(V, E)$ where the set of mutants $Y$ have fitness $r$, let $r_{v, Y}=r$ if $v \in Y$ and $r_{v, Y}=1$, otherwise. We define the continuous-time version of the Moran process on a digraph $G=(V, E)$ as follows. Starting in configuration $\widetilde{Y}[t]$ at time $t$, each vertex $v$ waits for a period of time before reproducing. This period of time is chosen, independently of other vertices, according to an exponential distribution with parameter $r_{v, \widetilde{Y}[t]}$. Therefore, the probability that two vertices reproduce at once is zero. Suppose that the first vertex to reproduce after time $t$ is vertex $v$, at time $t+\tau$. As in the discrete-time version of the process, an out-neighbour $w$ of $v$ is chosen u.a.r. and the new configuration $\widetilde{Y}[t+\tau]$ is $\widetilde{Y}[t] \cup\{w\}$ if $v$ is a mutant and $\widetilde{Y}[t] \backslash\{w\}$, otherwise.

From the definition of the exponential distribution, it is clear that the probability that a particular vertex $v$ is the next to reproduce, from configuration $\widetilde{Y}[t]$, is $r_{v, \widetilde{Y}[t]} / W_{|\widetilde{Y}[t]|^{\circ}}$ Thus, the Moran process (as generalised by Lieberman et al.) is recovered by taking the sequence of configurations each time a vertex reproduces ${ }^{1}$ so the continuous-time process is a faithful simulation of the discrete version.

Using the continuous-time version of the process, we are able to prove the coupling lemma (Lemma 5), Theorem 6 and its corollaries, as stated in Section 1.1.3.

\section{$4 \quad$ Regular Digraphs}

This section provides upper and lower bounds on the absorption time of the Moran process on regular digraphs. Clearly, the bounds on expected absorption time of undirected graphs from [10] do not apply to digraphs since [10, Theorem 7] gives a polynomial upper bound but our Theorem 4 shows that process takes exponential time on some strongly connected digraphs. Since we will be discussing both undirected graphs and digraphs in this section,

1 This is closely related to the jump chain, which is defined to be the discrete-time chain whose successive states are the states $\widetilde{Y}[t]$ for the successive times $t$ immediately after the state changes. Thus, the jump chain is the chain of "active" steps of the discrete-time Moran process (see Section 4.2). 
we start by observing as Proposition 9 that [10, Theorem 7] can be improved to give an $O\left(n^{3}\right)$ bound on regular undirected graphs. This is certainly not tight (see below) but it is a natural place to begin.

- Proposition 9. The expected absorption time of the Moran process on a connected $\Delta$-regular $n$-vertex undirected graph is at most $\frac{r}{r-1} n^{2} \Delta$.

\subsection{Definitions}

We will use the following standard Markov chain definitions. For more detail, see, for example, [24]. We use $\left(X_{t}\right)_{t \geq 0}$ to denote a discrete-time Markov chain $\mathcal{M}$ with finite state space $\Omega$ and transition matrix $P . T_{k}=\inf \left\{t \geq 1 \mid X_{t}=k\right\}$ is the first passage time for visiting state $k$ (not counting the initial state $X_{0}$ ). The time spent in state $i$ between visits to state $k$ is

$$
\gamma_{i}^{k}=\sum_{t=0}^{T_{k}-1} 1_{X_{t}=i}, \text { where } X_{0}=k .
$$

The chain is irreducible if, for every pair of states $(i, j)$ there is some $t \geq 0$ such that $\operatorname{Pr}\left(X_{t}=j \mid X_{0}=i\right)>0$. Since $\Omega$ is finite, this implies that the chain is recurrent, which means that, for every state $i \in \Omega, \operatorname{Pr}\left(X_{t}=i\right.$ for infinitely many $\left.t\right)=1$. We use the following proposition, which (up to minor notational differences) is the special case of $[24$, Theorem 1.7.6] for finite state spaces.

Proposition 10. Let $\mathcal{M}$ be an irreducible discrete-time Markov chain with finite state space $\Omega=\{0, \ldots, \omega-1\}$ and transition matrix $P$. For $k \in \Omega$, let $\lambda=\left(\lambda_{0}, \ldots, \lambda_{\omega-1}\right)$ be a vector of non-negative reals with $\lambda_{k}=1$, satisfying $\lambda P=\lambda$. Then for every $j \in \Omega, E\left[\gamma_{j}^{k}\right]=\lambda_{j}$.

\subsection{Active Steps and Absorption Time}

We fix $r>1$ and study the Moran process on a strongly connected $\Delta$-regular $n$-vertex digraph $G=(V, E)$ with $n>1$. An active step is one at which the number of mutants changes. As explained in the introduction, the number of mutants after each active step corresponds to a one-dimensional random walk on $\{0, \ldots, n\}$ which starts at state 1 , absorbs at 0 and $n$, and has upwards drift $p=\frac{r}{r+1}$.

To derive the properties that we need, we consider a Markov chain $\mathcal{M}$ with state space $\Omega=\{0, \ldots, n+1\}$. The non-zero entries of the transition matrix $P$ of $\mathcal{M}$ are $P_{0, n+1}=P_{n, n+1}=P_{n+1,1}=1$ and, for $1 \leq i \leq n-1, P_{i, i+1}=p$ and $P_{i, i-1}=1-p$. Starting from state 1 , the chain simulates the one-dimensional walk discussed above. State $n+1$ is visited immediately after an absorbing state of the random walk is reached. From state $n+1$, the chain goes back to state 1 and repeats the random walk. We establish the following key property of $\mathcal{M}$, which is proved in the full version.

- Lemma 11. Let $f=\left(r^{n}-r^{n-1}\right) /\left(r^{n}-1\right)$. Define the vector $\lambda=\left(\lambda_{0}, \ldots, \lambda_{n+1}\right)$ as follows.

$$
\begin{aligned}
& \lambda_{0}=1-f, \quad \lambda_{n}=f, \quad \lambda_{n+1}=1, \\
& \lambda_{j}=(1+r)(1-f)\left(r^{n}-r^{j}\right) /\left(r^{n}-r\right), \text { for } 1 \leq j \leq n-1 .
\end{aligned}
$$

Then, for every $j \in \Omega, E\left[\gamma_{j}^{n+1}\right]=\lambda_{j}$.

It is well known [17], and an easy consequence of Lemma 11, that $f$ is the fixation probability of the Moran process on a regular graph. We use the following corollary; upper bound is because $r^{n}-r^{j} \leq r^{n}-1$ and the lower bound is because $E\left[\gamma_{j}^{n+1}\right]$ is minimised at $j=n-1$. 
- Corollary 12. For all $j \in\{1, \ldots, n-1\}, 1-\frac{1}{r^{2}} \leq E\left[\gamma_{j}^{n+1}\right] \leq 1+\frac{1}{r}$.

Now, let the Moran process on a digraph $G$ be $\left(Y_{t}\right)_{t \geq 1}$. For each state $S$, let $p(S)=$ $\operatorname{Pr}\left(Y_{t+1} \neq S \mid Y_{t}=S\right)$ and let $\mu(S)=\inf \left\{t \geq 1 \mid Y_{t+1} \neq S, Y_{1}=S\right\}$. The random variable $\mu(S)$ is the number of steps for which the process stays in state $S$ after arriving there. It is geometrically distributed with parameter $p(S)$, so $E[\mu(S)]=1 / p(S)$. Let $\tau_{1}=1$. For $j>1$, let $\tau_{j}=\inf \left\{t>\tau_{j-1} \mid Y_{t} \neq Y_{t-1}\right\}$. The values $\tau_{2}, \tau_{3}, \ldots$ are the active steps and $\left(Y_{\tau_{j}}\right)_{j \geq 1}$ is the Moran process with the repeated states omitted. The time spent in state $Y_{\tau_{j-1}}$ is $\tau_{j}-\tau_{j-1}$ and the absorption time $T_{\mathrm{A}}$ of the Moran process is the sum of these times. If we take $X_{0}=n+1, T_{\mathrm{A}}$ is also $\tau_{T_{n+1}-1}$, where $T_{n+1}$ is the first passage time of state $n+1$ in the process $\left(X_{t}\right)_{t \geq 0}$, above. For convenience, let $\ell=T_{n+1}-1$.

To derive upper and lower bounds for $E\left[T_{\mathrm{A}}\right]$, we break the sum into pieces. For $k \in[n-1]$, let $T_{\mathrm{A}, k}$ be the total number of steps spent in states with $k$ mutants before absorption. $T_{\mathrm{A}, k}=\sum_{j=2}^{\ell} \Psi_{k, j}$, where $\Psi_{k, j}$ is geometrically distributed with parameter $p\left(Y_{\tau_{j-1}}\right)$ if $\left|Y_{\tau_{j-1}}\right|=k$ and $\Psi_{k, j}=0$, otherwise. Then $T_{\mathrm{A}}$ is distributed as $\sum_{k=1}^{n-1} T_{\mathrm{A}, k}$. To derive the bounds, let $p_{k}^{+}=\max \{p(S)|| S \mid=k\}$ and $p_{k}^{-}=\min \{p(S)|| S \mid=k\}$. Let $T_{\mathrm{A}, k}^{+}=\sum_{j=2}^{\ell} \Psi_{k, j}^{+}$ where $\Psi_{k, j}^{+}$is geometrically distributed with parameter $p_{k}^{-}$if $\left|Y_{\tau_{j-1}}\right|=k$ and $\Psi_{k, j}^{+}=0$, otherwise. Let $T_{\mathrm{A}, k}^{-}=\sum_{j=2}^{\ell} \Psi_{k, j}^{-}$where $\Psi_{k, j}^{-}$is geometrically distributed with parameter $p_{k}^{+}$ if $\left|Y_{\tau_{j-1}}\right|=k$ and $\Psi_{k, j}^{-}=0$, otherwise. Then by stochastic domination for the geometric distribution, $\sum_{k=1}^{n-1} E\left[T_{\mathrm{A}, k}^{-}\right] \leq E\left[T_{\mathrm{A}}\right] \leq \sum_{k=1}^{n-1} E\left[T_{\mathrm{A}, k}^{+}\right]$.

Thus, we have upper and lower bounds for $E\left[T_{\mathrm{A}}\right]$ as sums of random numbers (the $\gamma_{k}^{n+1}$ ) of geometric random variables, each having parameter $1 / p_{k}^{+}$or $1 / p_{k}^{-}$for some $k$. Corollary 12 shows that $E\left[\gamma_{k}^{n+1}\right]$ is finite; all the parameters $1 / p_{k}^{+}$and $1 / p_{k}^{-}$are also finite. Wald's equality and Corollary 12 give us the following theorem.

- Theorem 13. $\left(1-\frac{1}{r^{2}}\right) \sum_{k=1}^{n-1}\left(1 / p_{k}^{+}\right) \leq E\left[T_{A}\right] \leq\left(1+\frac{1}{r}\right) \sum_{k=1}^{n-1}\left(1 / p_{k}^{-}\right)$.

\subsection{Upper and Lower Bounds}

Observe that, if $|S|=k$ then $p(S)=\frac{r m_{S}^{+}}{W_{k} \Delta}+\frac{m_{S}^{-}}{W_{k} \Delta}$, so $\frac{1}{p(S)}=\frac{W_{k} \Delta}{r m_{S}^{+}+m_{S}^{-}}$. From this and Theorem 13, we get the following, which allows us to prove Theorem 1.

- Corollary 15. The expected absorption time of the Moran process on a strongly connected $\Delta$-regular $n$-vertex digraph $G$ is at least $\left(1-\frac{1}{r^{2}}\right) W_{1} \Delta M$ and at most $\left(1+\frac{1}{r}\right) W_{n} \Delta M$, where $M$ denotes $\sum_{k=1}^{n-1} 1 / \min \left\{r m_{S}^{+}+m_{S}^{-}|| S \mid=k\right\}$.

- Theorem 1. The expected absorption time of the Moran process on a strongly connected $\Delta$-regular $n$-vertex digraph $G$ is at least $\left(\frac{r-1}{r^{2}}\right) n H_{n-1}$ and at most $n^{2} \Delta$.

Proof. If $|S|=k$ then we have $r m_{S}^{+}+m_{S}^{-} \leq(r+1) k \Delta$, which, with Corollary 15 , establishes the lower bound. If a digraph is strongly connected, then $m_{S}^{+}, m_{S}^{-} \geq 1$ when $1 \leq|S| \leq n-1$ so $r m_{S}^{+}+m_{S}^{-} \geq r+1$. This, together with Corollary 15, establishes the upper bound.

Note that the upper bound in Theorem 1 generalises the one given in Proposition 9 to the directed case. The following observations follow from special cases of Corollary 15. All match the corresponding bounds from Theorem 1 up to a constant factor (which may depend on $r$ but not on $n$ ).

- Observation 16. Let $G$ be the undirected clique $K_{n}$ (which is $\Delta$-regular with $\Delta=n-1$ ). For $S$ of size $k, m_{S}^{+}=m_{S}^{-}=k(n-k)$, so Corollary 15 shows that the absorption time is at 
most

$$
n \sum_{k=1}^{n-1} \frac{n-1}{k(n-k)} \leq n \sum_{k=1}^{n-1} \frac{n-k}{k(n-k)}+n \sum_{k=1}^{n-1} \frac{k}{k(n-k)} \leq 2 n H_{n-1} .
$$

- Observation 17. Let $G$ be the undirected cycle $C_{n}$ (which is $\Delta$-regular with $\Delta=2$ ). Since the process starts with a single mutant, it is easy to see that the set of mutant vertices must be connected, if it is non-empty. Therefore, $m_{S}^{+}=m_{S}^{-}=2$ for any non-trivial state $S$ that is reachable from the initial configuration, so the absorption time is at least $\left(1-\frac{1}{r^{2}}\right) \frac{2 n}{r+1} \sum_{k=1}^{n-1} \frac{1}{2}=\Omega\left(n^{2}\right)$.

- Observation 18. Let $G$ be the directed $n$-vertex cycle (which is $\Delta$-regular with $\Delta=1$ ). Again, the mutants remain connected; in this case $m_{S}^{+}=m_{S}^{-}=1$ for any non-trivial reachable $S$ so the absorption time is at least $\left(1-\frac{1}{r^{2}}\right) \frac{n}{r+1} \sum_{k=1}^{n-1} \frac{1}{2}=\Omega\left(n^{2}\right)$.

\subsection{Better Upper Bounds for Undirected Graphs via Isoperimetric Numbers}

For an undirected graph $G$ and $S \subseteq V(G)$, let $\partial S$ be the set of edges between $S$ and $V(G) \backslash S$. Then $m_{S}^{+}=m_{S}^{-}=|\partial S|$. The isoperimetric number of $G$ was defined by Buser [7] as

$$
i(G)=\min \{|\partial S| /|S||S \subseteq V(G), 0<| S|\leq| V(G) \mid / 2\} .
$$

The quantity $i(G)$ is a discrete analogue of the Cheeger isoperimetric constant. For graphs with good expansion, Theorem 3 improves the upper bound from Theorem 1, as discussed in Section 1.1.1.

\subsection{Families for Which the Upper Bound is Optimal}

For every fixed $\Delta>2$, we construct an infinite family of connected, $\Delta$-regular undirected graphs for which the upper bound in Theorem 1 is optimal, up to a constant factor (which may depend upon $r$ and $\Delta$ but not on $n$ ).

To do this, we define the graph $H_{\Delta}$ to be $K_{\Delta-2, \Delta-1}$ with the addition of edges forming a cycle on the side with $\Delta-1$ vertices. Now, let $G_{\ell, \Delta}$ be the $\Delta$-regular graph formed from a cycle $C=x_{1} \ldots x_{\ell} x_{1}$ and $\ell$ disjoint copies of $H_{\Delta}$ by adding an undirected edge between $x_{i}$ and each of the vertices of degree $\Delta-1$ in the $i$ 'th copy of $H_{\Delta}$, for each $i \in[\ell]$. Note that $\left|V\left(G_{\ell, \Delta}\right)\right|=2 \ell(\Delta-1)$. Intuitively, the limiting factor for the propagation of mutants through the graph is that is connected only through the cycle $C$ and absorption time on cycles is quadratic (Observation 17; with some work and careful use of stochastic dominations, this can be formalised into a proof of the following theorem.

- Theorem 19. For $r>1$ and sufficiently large $\ell$ (with respect to $r$ ), the expected absorption time of the Moran process on $G_{\ell, \Delta}$ exceeds $\frac{1}{2 r}\left(1-\frac{1}{r}\right) \ell^{2}$.

Thus, the $O\left(n^{2}\right)$ upper bound of Theorem 1 is tight up to a constant factor (which may depend on $r$ and $\Delta$, but not on $n$ ). For given $r$ and $\Delta$, let $\mathcal{G}$ be the class of all graphs $G_{\ell, \Delta}$ with $\ell$ large enough for Theorem 19 to apply: this proves Theorem 2.

\section{General Digraphs}

Fix $r>1$ and let $\varepsilon_{r}=\min (r-1,1)$. Theorem 7 of $[10]$ shows that the expected absorption time of the Moran process on a connected $n$-vertex undirected graph is at most $\frac{r}{r-1} n^{4}$. 


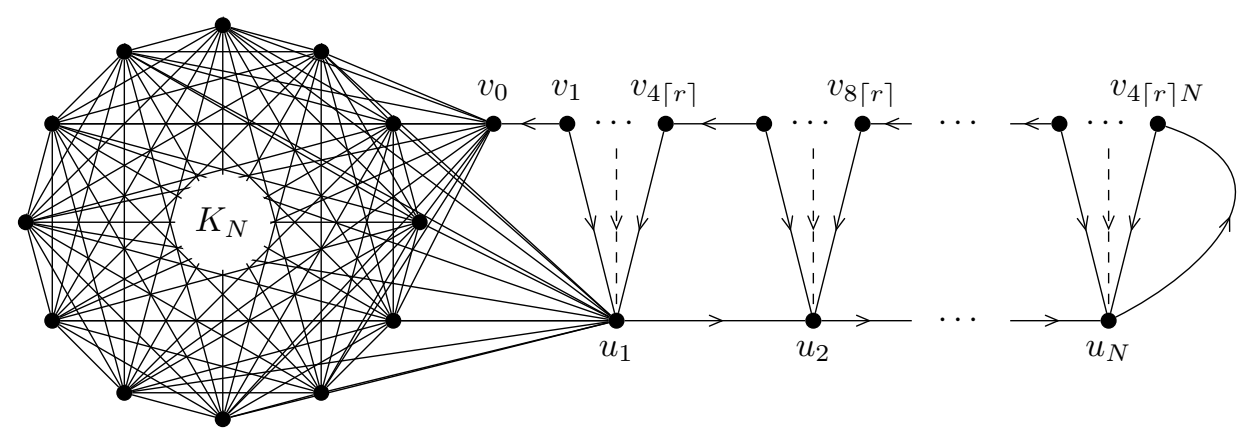

Figure 1 The graph $G_{r, N}$. The edges within the clique are bidirectional; $v_{0}$ sends a directed edge to every vertex in the clique and $u_{1}$ receives one from each. Other edges are directed as indicated.

Theorem 1 shows that the expected absorption time on a strongly connected $\Delta$-regular digraph is at most $n^{2} \Delta$. In contrast, we exhibit an infinite family of strongly connected digraphs such that the expected absorption time on an $n$-vertex graph from the family is $2^{\Omega(n)}$.

Let $G_{r, N}$ be the disjoint union of the complete graph $K_{N}$ (with bidirectional edges), a directed path $P=u_{1} \ldots u_{N}$ and a directed path $Q=v_{4\lceil r\rceil N} \ldots v_{0}$, along with the directed edge $\left(u_{N}, v_{4\lceil r\rceil N}\right)$ and the directed edges $\left(x, u_{1}\right)$ and $\left(v_{0}, x\right)$ for every $x \in K_{N}$ and $\left(v_{4\lceil r\rceil(i-1)+j}, u_{i}\right)$ for each $i \in[N], j \in[4\lceil r\rceil]$ (see Figure 1).

Intuitively, the Moran process on $G_{r, N}$ behaves as follows. With probability close to $\frac{1}{4[r]+2}$, the initial mutant is in the clique. Since there are $N(N-1)$ directed edges within the clique and only $2 N$ between it and the rest of the graph, it is reasonable to expect that the behaviour of the mutants within the clique is close to the behaviour of mutants in a complete graph, which is a one-dimensional random walk. The main challenge is to show that, conditioned on the initial mutant being in the clique, enough reproductions happen in the clique in the first $N^{3}$ steps of the process that the probability of the clique becoming at least half full of mutants within the first $N^{3}$ steps of the process is at least $\varepsilon_{r} / 8$. From this state, we show that both fixation and extinction are likely to be slow.

Fixation cannot occur unless all vertices in $P$, the first part of the path, become mutants. It is relatively straightforward to show that, conditioned on the initial mutant being in the clique, the probability of $P$ filling with mutants within the first $T^{*}(N)$ steps is also at most $\varepsilon_{r} / 32$. The argument proceeds by a stochastic domination. We allow only the mutant in $P$ that is farthest from the clique to be replaced by a non-mutant, so the mutants in $P$ occupy an initial segment of the path. The number of mutants in $P$ behaves as a random walk with downward drift, since the "mutant frontier" in $P$ is more likely to be pushed back by the edges from $Q$ than it is to move forwards.

On the other hand, extinction cannot happen while there are still mutants in the clique. As observed in Section 1.1.1, the number of mutants in a complete graph is a random walk with upward drift $r$. We may approximate the behaviour of the mutants in the clique by a one-dimensional random walk with upward drift $r$ that has a non-absorbing barrier at $N$. (Since the clique is not the whole graph, filling the clique with mutants does not correspond to absorption of the process.) Such a walk is expected to take exponentially long to return to zero. This allows us to show that, conditioned on the initial mutant being in the clique and the clique reaching $N / 2$ mutants by step $N^{3}$, the probability that the number of mutants in the clique reaches zero by step $T^{*}(N)$ is at most $\varepsilon_{r} / 32$, where $T^{*}(N)=\left\lfloor\varepsilon_{r}\left(2^{N}-1\right) / 32\right\rfloor$. 
Therefore, the conditional probability of extinction within the first $T^{*}(N)$ steps is also at most $\varepsilon_{r} / 32$.

Combining these results, and using Markov's inequality, we obtain the lower bound on the expected absorption time of the Moran process on $G_{r, N}$, given by Theorem 4 of Section 1.1.2.

\section{References}

1 Tibor Antal and István Scheuring. Fixation of strategies for an evolutionary game in finite populations. Bulletin of Mathematical Biology, 68(8):1923-1944, 2006.

2 Chalee Asavathiratham, Sandip Roy, Bernard Lesieutre, and George Verghese. The influence model. IEEE Control Systems, 21(6):52-64, 2001.

3 Eli Berge. Dynamic monopolies of constant size. Journal of Combinatorial Theory, Series B, 83(2):191-200, 2001.

4 Béla Bollobás. The isoperimetric number of random regular graphs. European J. Combin., 9(3):241-244, 1988.

5 M. Broom, C. Hadjicrysanthou, and J. Rychtár. Evolutionary games on graphs and the speed of the evolutionary process. Proceedings of the Royal Society A, 466(2117):1327-1346, 2010 .

6 M. Broom and J. Rychtár. An analysis of the fixation probability of a mutant on special classes of non-directed graphs. Proceedings of the Royal Society A, 464(2098):2609-2627, 2008.

7 Peter Buser. Cubic graphs and the first eigenvalue of a Riemann surface. Math. Z., 162(1):87-99, 1978.

8 M. Cemil Azizoğlu and Ömer Eğecioğlu. The bisection width and the isoperimetric number of arrays. Discrete Appl. Math., 138(1-2):3-12, 2004.

9 Josep Díaz, Leslie Ann Goldberg, George B. Mertzios, David Richerby, Maria J. Serna, and Paul G. Spirakis. On the fixation probability of superstars. Proceedings of the Royal Society A, 469(2156):20130193, 2013.

10 Josep Díaz, Leslie Ann Goldberg, George B. Mertzios, David Richerby, Maria J. Serna, and Paul G. Spirakis. Approximating fixation probabilities in the generalized Moran process. Algorithmica, to appear.

11 Josep Díaz, Leslie Ann Goldberg, David Richerby, and Maria J. Serna. Absorption time of the moran process. CoRR, abs/1311.7631, 2013.

12 Herbert Gintis. Game Theory Evolving: A Problem-Centered Introduction to Modeling Strategic Interaction. Princeton University Press, 2000.

13 G. R. Grimmett and D. R. Stirzaker. Probability and Random Processes. Oxford University Press, 3rd edition, 2001.

14 B. Houchmandzadeh and M. Vallade. The fixation probability of a beneficial mutation in a geographically structured population. New Journal of Physics, 13:073020, 2011.

15 A. Jamieson-Lane and C. Hauert. Fixation probabilities on superstars, revisited and revised. ArXiv 1312.6333, 2013.

16 David Kempe, Jon Kleinberg, and Éva Tardos. Maximizing the spread of influence through a social network. In Proc. 9th ACM International Conference on Knowledge Discovery and Data Mining, pages 137-146. ACM, 2003.

17 Erez Lieberman, Christoph Hauert, and Martin A. Nowak. Evolutionary dynamics on graphs. Nature, 433(7023):312-316, 2005.

18 Thomas M. Liggett. Stochastic Interacting Systems: Contact, Voter and Exclusion Processes. Springer, 1999. 
19 George B. Mertzios, Sotiris E. Nikoletseas, Christoforos Raptopoulos, and Paul G. Spirakis. Natural models for evolution on networks. Theoretical Computer Science, 477:76-95, 2013.

20 George B. Mertzios and Paul G. Spirakis. Strong bounds for evolution in networks. In Proc. 40th International Colloquium on Automata, Languages and Programming (ICALP 2013), volume 7966 of $L N C S$, pages 657-668. Springer, 2013.

21 Bojan Mohar. Isoperimetric numbers of graphs. Journal of Combinatorial Theory, Series B, 47(3):274-291, 1989.

22 P. A.P. Moran. Random processes in genetics. Proceedings of the Cambridge Philosophical Society, 54(1):60-71, 1958.

$23 \mathrm{Pu}-$ Yan Nie and Pei-Ai Zhang. Fixation time for evolutionary graphs. International Journal of Modern Physics B, 24(27):5285-5293, 2010.

24 James R. Norris. Markov Chains. Cambridge University Press, 1998.

25 Paulo Shakarian and Patrick Roos. Fast and deterministic computation of fixation probability in evolutionary graphs. In Proc. 6th International Conference on Computational Intelligence and Bioinformatics, pages 753-012. ACTA Press, 2011.

26 Paulo Shakarian, Patrick Roos, and Anthony Johnson. A review of evolutionary graph theory with applications to game theory. Biosystems, 107:66-80, 2012.

27 Christine Taylor, Drew Fudenberg, Akira Sasaki, and Martin A. Nowak. Evolutionary game dynamics in finite populations. Bulletin of Mathematical Biology, 66(6):1621-1644, 2004. 